\title{
DYNAMICAL INSTABILITIES, MANIFOLDS, AND LOCAL LYAPUNOV SPECTRA FAR FROM EQUILIBRIUM
}

\author{
WM. G. HOOVER ${ }^{1,2}$, CAROL G. HOOVER ${ }^{2}$, H. A. POSCH \\ I Department of Applied Science, University of California at Davis, \\ Lawrence Livermore National Laboratory, Livermore 94550, California, USA \\ 2 Methods Development Group, Department ofMechanical Engineering, \\ Lawrence Livermore National Laboratory, Livermore 94550, California, USA \\ 3 Institut für Experimentalphysik, Universität Wien, \\ Boltzmanngasse 5, A-1090 Wien, Austria
}

\begin{abstract}
We consider an harmonic oscillator in a thermal gradient far from equilibrium. The motion is made ergodic and fully time-reversible through the use of two thermostat variables. The equations of motion contain both linear and quadratic terms. The dynamics is chaotic. The resulting phase-space distribution is not only complex and multifiactal, but also ergodic, due to the time-reversibility property. We analyze dynamical time series in two ways. We describe local, but comoving, singularities in terms of the "local Lyapunov spectrum" $\{\lambda\}$. Local singularities at a particular phase-space point can alternatively be described by the local eigenvalues and eigenvectors of the "dynamical matrix" $D \equiv \partial v / \partial r \equiv \nabla \nu . D$ is the matrix of derivatives of the equations of motion $\dot{r}=\boldsymbol{v}(r)$. We pursue this eigenvalue-eigenvector description for the oscillator. We find that it breaks down at a dense set of singular points, where the four eigenvectors span only a three-dimensional subspace. We believe that the concepts of stable and unstable global manifolds are problematic for this simple nonequilibrium system.
\end{abstract}

PACS numbers: 05.45.A, 05.10, 07.05.T

\section{INTRODUCTION - CHARACTERIZING CHAOS}

The stability of a phase-space "flow" $v(r)$ with one or more "fixed points" - points where the phase-space velocity $\mathrm{v}$ vanishes - is most naturally expressed in terms of the flow's "dynamical matrix" $D \equiv \nabla v$. Positive eigenvalues of this matrix correspond to exponential growth. Negative ones indicate decay, toward the fixed point. Complex eigenvalues can also occur. For example, the undamped harmonic oscillator, with its phase-space flow velocity

$$
\nu \equiv\{\dot{q}, \dot{p}\}=\{+p,-q\} \rightarrow D=\left(\begin{array}{cc}
0 & +1 \\
-1 & 0
\end{array}\right) .
$$

has eigenvalues $\{ \pm i\}$ corresponding to oscillation about the fixed-point origin $\{q, p\}=\{0,0\}$. For more complicated systems - a pendulum is the usual example - it is usual to define stable and unstable "manifolds", unions of those phase-space directions which correspond to exponential decay and to exponential growth, respectively [1]. In even more complicated situations, including 
the thermally-driven oscillator which we consider in the present work, there may be no stable fixed points. Even so, it is still possible to evaluate the eigenvalues of $\nabla v$. Here we follow this approach for an oscillator, bound to the origin at $q=0$, but moving in a nonuniform thermal bath, with $T_{q} \equiv 1+\varepsilon \tanh q$. The temperature $T_{q}$ varies from $1-\varepsilon$ to $1+\varepsilon$, with a maximum temperature gradient of $\varepsilon$ at $q=0$. The corresponding dynamics includes both quadratic and linear forces, as is described in Section II. As a result, the dynamical matrix $D$ varies from point to point. Because the eigenvalues of $D$ are typically complex, the eigenvectors are seldom orthogonal.

Lyapunov-unstable systems, like the nonequilibrium oscillator, are characterized by trajectory perturbations which grow as $e^{\lambda t}$. The growth can be described by the "global Lyapunov spectrum". This spectrum of exponents gives the long-time-averaged rates of growth and decay governing.nearby trajectories. The local, or instantaneous, Lyapunov spectrum $\{\lambda\}$ gives more details. It describes the linearized deformation of an infinitesimal phase-space ball, or hypersphere [2-4] centered on a comoving origin which tracks a phase-space trajectory. The individual local Lyapunov exponents, at a particular phase-space location along the trajectory, give the orthogonal growth rates of the ball, in the comoving and corotating coordinate system. The long-time-averaged values of these local exponents comprise the conventional (time-independent, or "global") Lyapunov spectrum In a four-dimensional phase-space, for example, the longtime-averaged spectrum has four exponents:

$$
\langle\{\lambda\}\rangle=\left\langle\left\{\lambda_{1}, \lambda_{2}, \lambda_{3}, \lambda_{4}\right\}\right\rangle
$$

Spectra, both local and global, have been computed for a wide variety of few-body and many-body problems [2-6], Evidently these local exponents differ from Gaspard's "local expansion rates". At present there is no robust algorithm available for computing the latter [7].

Quite generally, as follows from their definition, the summed-up local Lyapunov spectrum gives the instantaneous rate with which an infinitesimal phase volume $\otimes$ changes with time in response to the equations of motion: $\sum \lambda \equiv \dot{\otimes} / \otimes$. Gibbs called such a comoving volume an "extension in phase". For particular nonequilibrium forms of dynamics (Nosé-Hoover dynamics is the best example), the local and global spectra can additionally be related to the dissipative entropy production:

$$
\sum \lambda \equiv-\dot{S} / k \rightarrow \sum\langle\lambda\rangle \equiv\langle-\dot{S} / k\rangle
$$

where $k$ is Boltzmann's constant. An overall positive entropy production corresponds to a long-time-averaged collapse of the phase volume to a "strange attractor", an object with (information) dimensionality strictly less than that of the full phase space. A persistent negative entropy production is not possible. It would lead to a diverging extension in phase, $\otimes \rightarrow \infty$, and to a violation of the second law of thermodynamics [2,3], Both the macroscopic entropy production and the dimensionality of the microscopic phase space distribution have been analyzed for a variety of diffusive, viscous, and heat-conducting flows [2-4].

The fractal nature of the nonequilibrium distributions, even for time-reversible flows, was controversial as recently as 1990 [8], The fractals are widely accepted now. They have motivated 
attempts to link the distribution to the local Lyapunov spectrum, usually smoothed over a time interval of the order of the Green-Kubo relaxation time [9], The fractal nature of the distribution functions seems to us particularly difficult to reconcile with the notion of stable and unstable manifolds so familiar from studies of systems with stable fixed points [1], We believe that the particular model detailed in the following section is specially useful for understanding and interpreting topological ideas. Though it would be very useful to do so, we have so far not succeeded in formulating the Green-Kubo linear-response theory for the present model.

The equilibrium properties of this chaotic oscillator model were studied recently and revealed that the variation of the local Lyapunov exponents in phase space has an interesting fractal character [6], Though the local Lyapunov exponents are indeed well-defined "point functions", with definite values at any given phase-space location, they are singular everywhere. These ubiquitous singularities reflect the exponentially-large differences between the past histories of any two neighboring trajectories. In the present work we examine the local dynamical matrix $D$ as well as the local Lyapunov spectrum for a nonequilibrium oscillator model. The dynamical matrix is itself a pointfunction in phase space, with the forward and backward time directions simply related, as is detailed in Section V. By contrast, the local Lyapunov spectrum, which reflects the trajectory's past history in selecting the directions along which growth and decay are measured, reveals a time-symmetry breaking which makes a straightforward analysis difficult. By emphasizing the time-symmetry properties of the dynamical matrix $D$ and the breaking of symmetry in the Lyapunov spectrum - by comparing local spectra in the two time directions - we investigate the usefulness of the spectrum to the task of describing nonequilibrium distribution functions. Our conclusions make up the final section.

\section{NONEQUILIBRIUM OSCILLATOR MODEL}

Our nonequilibrium oscillator, with coordinate $q$ and momentum $p=\dot{q}$, is controlled by two thermostat variables $\xi$ and $\xi$ [10], The dynamics takes place in a four-dimensional phase space $\{q, \mathrm{p}, \zeta, \xi\}$. The equations of motion are as follows:

$$
\dot{q}=p ; \dot{p}=-q-\zeta p ; \dot{\zeta}=p^{2}-T_{q}-\xi \zeta ; \dot{\xi}=\zeta^{2}-T_{q} .
$$

The dependence of temperature on the coordinate $q, T_{q}=1+\varepsilon \tanh q$, has to be sufficiently weak, with $0<\varepsilon<0.2632$. For stronger temperature gradients the motion collapses to a limit cycle and has no interesting chaotic properties. For a series of example motions, projected into the $q p$ plane, see Figure 1. There are two unstable fixed points, $(0,0, \pm 1, \mp 1)$.

The motion equations just given have a concealed dissipative character, despite their time reversibility. This causes the occupied phase volume to collapse to a strange attractor, $\otimes \rightarrow \mathbf{0}$. The rate of collapse is described exactly by Liouville's expression for the time-rate-of-change of the phase-space probability density $f(q, p, \xi, \xi)$ :

$$
\begin{gathered}
\dot{f} \equiv(\partial f / \partial t)+v \cdot \nabla f=-f(\nabla \cdot v)=-f \sum \lambda=f(\zeta+\xi) \\
\nabla \equiv\{\partial / \partial r\} ; r \equiv\{q, p, \zeta, \xi\} ; v \equiv\{\dot{q}, \dot{p}, \dot{\zeta}, \dot{\xi}\} .
\end{gathered}
$$



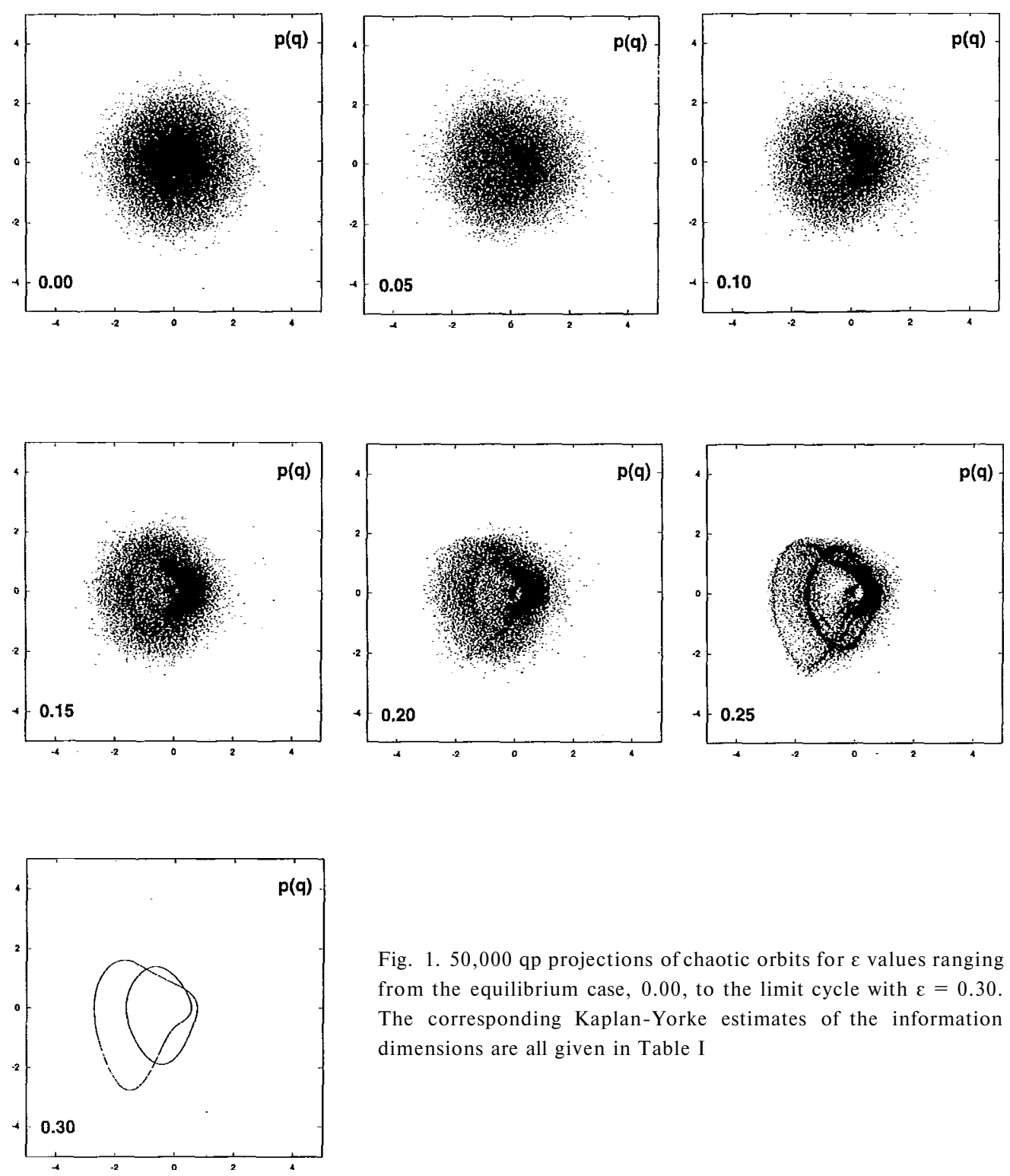

Fig. 1. 50,000 qp projections of chaotic orbits for $\varepsilon$ values ranging from the equilibrium case, 0.00 , to the limit cycle with $\varepsilon=0.30$. The corresponding Kaplan-Yorke estimates of the information dimensions are all given in Table I

In the simpler equilibrium case, with the temperature constant, $\varepsilon \equiv 0 \rightarrow T_{q}=T=1$, the stationary solution for $f$ is given by Gibbs' canonical distribution:

$$
f_{\text {equilibrium }} \propto e^{-\left(q^{2}+p^{2}+\zeta^{2}+\xi^{2}\right) / 2} .
$$

The form of this stationary distribution suggests an effective "Hamiltonian" $\mathcal{H}_{\mathrm{e}}$;

$$
\mathcal{H}_{\mathrm{e}} \equiv\left(q^{2}+p^{2}+\zeta^{2}+\xi^{2}\right) / 2 .
$$


The strong mixing properties induced by the quadratic forces in the equations of motion cause changes in the numerical value of $\mathcal{H}_{e}$ as time goes on, $\mathcal{H}_{e} \equiv-\zeta-\xi$ so that all the "energy shells" of constant $\mathcal{H}_{\mathrm{e}}$, with $0<\mathcal{H}_{\mathrm{e}}<\infty$, are included in the resulting distribution. In the nonequilibrium case, the change of this "energy" with respect to time depends upon the local temperature:

$$
\dot{\mathcal{H}}_{\mathrm{e}} \equiv-\dot{k} T_{q}(\zeta+\xi) \text {, }
$$

so that the control variables $(\zeta+\xi)=\sum \lambda$ play the rôle of an external entropy production, $S / k$.

\section{EIGENVALUES AND EIGENVECTORS OF THE DYNAMICAL MATRIX}

The dynamical matrix $D$ governs the time development of a small trajectory perturbation $\delta$ :

$$
\begin{gathered}
\{\dot{\delta}=D(r) \cdot \delta\} ; D(r) \equiv(\partial v / \partial r) \equiv(\partial \dot{r} / \partial r) ; \\
r=\{q, p, \zeta, \xi\} ; v=\{\dot{q}, \dot{p}, \dot{\zeta}, \dot{\xi}\} .
\end{gathered}
$$

For our flow, $D$ follows from the equations of motion:

$$
D=\left(\begin{array}{cccc}
0 & 1 & 0 & 0 \\
-1 & -\zeta & -p & 0 \\
-\varepsilon \cosh ^{-2} q & 2 p & -\xi & -\zeta \\
-\varepsilon \cosh ^{-2} q & 0 & 2 \zeta & 0
\end{array}\right)
$$
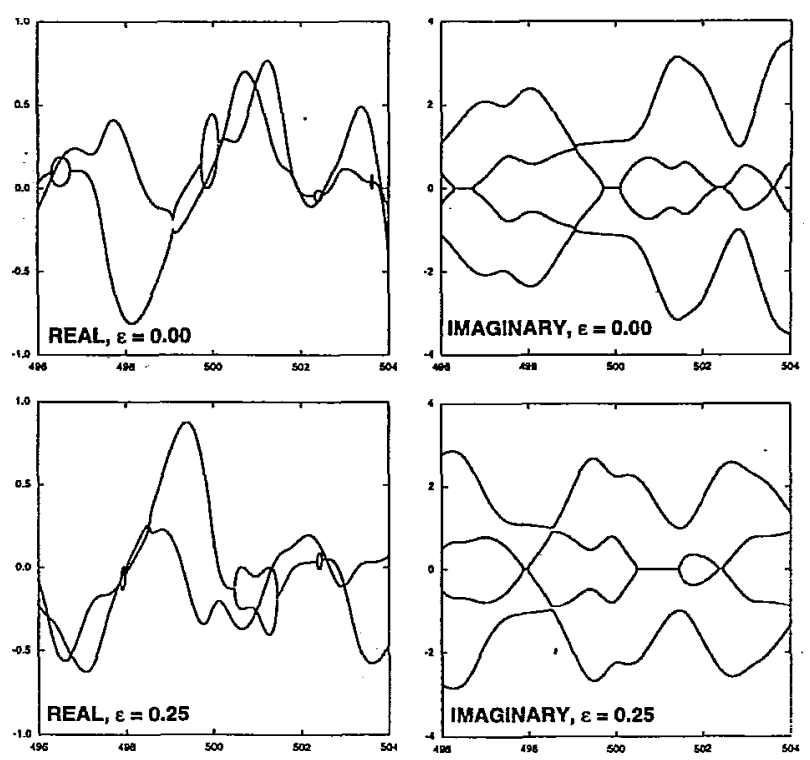

Fig. 2. Time variation of the real and imaginary components of the four Jacobian local eigenvalues for typical trajectory segments with $\varepsilon=0.00$ and $\varepsilon=0.25$. The imaginary components sometimes vanish, corresponding to the segments of the horizontal axis. At the ends of these segments two of the imaginary components appeal', or disappear. At the same times two of the eigenvalues, along with their eigenvectors, become exactly degenerate, so that a manifold description does not apply. Note that all the real components of the eigenvalues can have the same sign, either positive or negative 

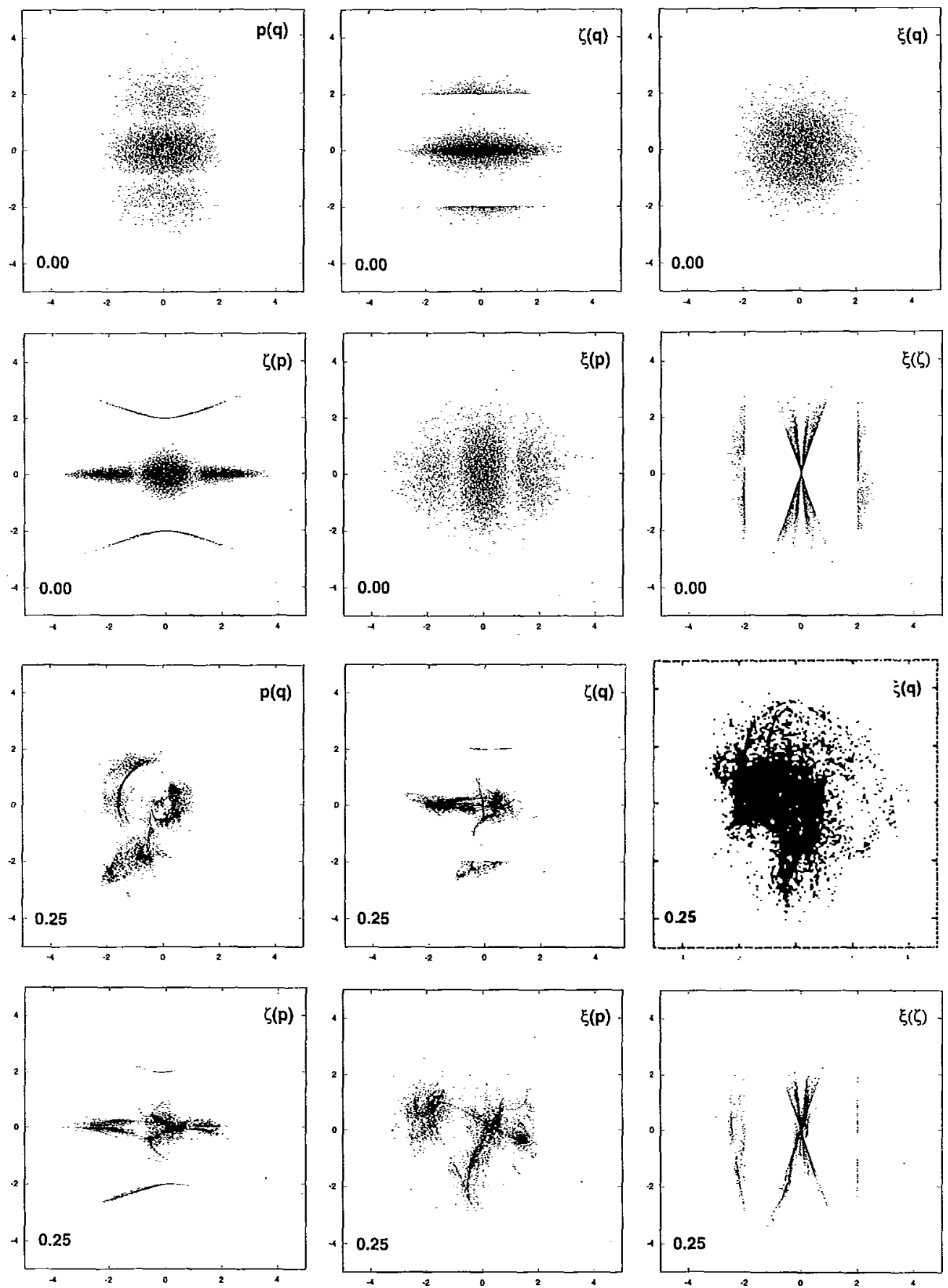

Fig. 3. Projections of singularity distributions in the $\{q, p, \xi, \xi\}$ phase space, for $\varepsilon=0.00$ (top half) and $\varepsilon=0.25$ (bottom half). The correlation-dimensions for these same data are analyzed in-Figure 4 
Because the dynamical matrix is neither symmetric nor Hermitian we cannot expect that the matrix has real eigenvalues. In fact, numerical investigation promptly reveals complex eigenvalues. The number of these complex eigenvalues jumps repeatedly between four (with two complex conjugate pairs) and two (with two exactly degenerate real roots at the time of the jumps). A typical case is illustrated in Figure 2. The complex roots reflect the tendency, locally only, of nearby points to follow a spiral track, toward or away from the trajectory, as is determined by the real parts of the complex roots. This spriraling tendency is only transient because there are no stable fixed points. $D$, together with its eigenvalues, changes according to the motion along the trajectory.

The jumps in complex eigenvalue numbers correspond to phase-space singularities. At these singularities two of the four eigenvalues, and also their eigenvectors, become degenerate. There can be only three independent eigenvectors at such singular points. At these points the three vectors can accordingly span only three of the four phase-space dimensions. The distribution of such singular points is evidently "dense" in a three-dimensional subspace of the four-dimensional embedding space. One-dimensional trajectories in the (four-dimensional) space cannot long avoid them. To visualize the distribution of the singularities, we generated the six projections shown in Figure 3. Their appearance suggests a fractal distribution.

In the equilibrium case, we carried out a quantitative investigation by computing the correlation dimension of the singularities. A plot of In $N(r)$, the (logarithm of the) number of pairs of singular points within a distance $r$, as a function of $\operatorname{In} r$ is given in Figure 4. The steepest part of the plot has a slope statistically indistinguishable from 3, suggesting that the equilibrium distribution of singularities is confined to a three-dimensional volume in the four-dimensional space. In the nonequilibrium case, the dimensionality is reduced, just as is that of the underlying attractor. Figure 4 shows the dependence of In $N(r)$ on In $r$ for $\varepsilon=0.25$. The correlation dimension in that case is approximately 2 .

Fig. 4. Variation of the relative number of singularity pairs $\mathrm{N}$ within a distance $\mathrm{r}$ for $\varepsilon=0.00$, and $\varepsilon=0.25$. The data represent $20,000,000$ timesteps, yielding 18,989 and 18,059 singular points in the two cases. The maximum slopes indicate correlation dimensions of about 3 and 2 , respectively

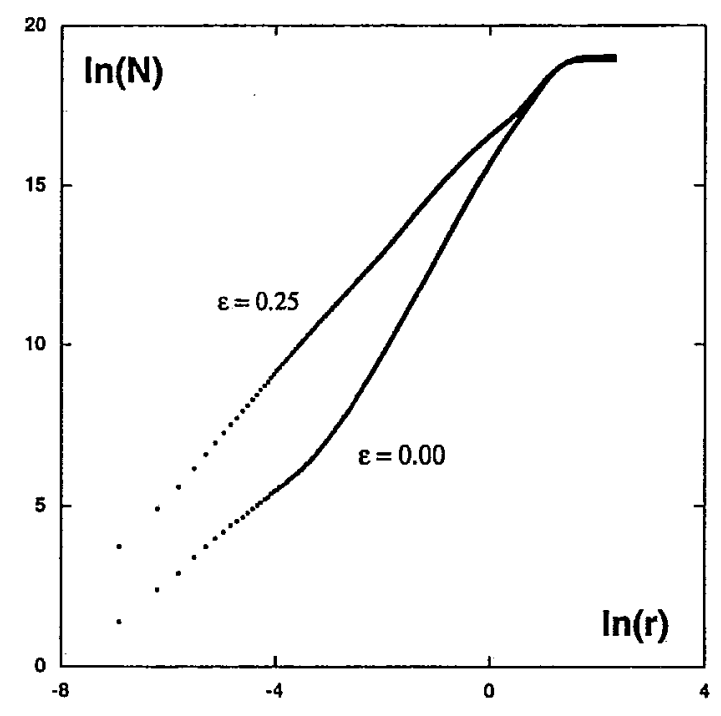


It is specially interesting to note that the real parts of all four eigenvalues can simultaneously all have the same sign. Whenever this happens the point at which the dynamical matrix is evaluated attracts or repels all nearby points. But because the attracting or repelling point is never fixed, this tendency is transient - otherwise the motion would have to stop or diverge! Thus a moving trajectory undergoes successive transient attractions and repulsions, resulting in a strange attractor, with no definite stable and unstable manifolds.

\section{COMPUTATION OF LOCAL LYAPUNOV SPECTRA}

The individual local Lyapunov exponents, at a particular phase-space location, give the orthogonal growth rates of a small phase-space hyperball $\otimes$, in a comoving and corotating coordinate system. The principal axes of the hyperball are parallel to the set of "offset vectors" $\{\delta\}$, which change with time. For an infinitesimal set of vectors $\{\delta\}$ the motion is determined by following the linearized equations of the underlying flow,

$$
\{\dot{\delta}=D(r) \cdot \delta\} ; \delta \equiv\{\delta q, \delta p, \delta \zeta, \delta \xi\}
$$

augmented to include the repeated orthogonalization and normalization of the "offset vectors" linking a central "reference trajectory" to nearby "satellite" trajectories [11], The orthogonalization can be carried out continuously, using Lagrange multipliers, or by discrete rescaling steps. Both approaches have been applied successfully [2-6].

For the present model, as the maximum temperature gradient e increases, the character of the spectrum,

$$
\langle\{\lambda\}\rangle=\left\langle\left\{\lambda_{1}, \lambda_{2}, \lambda_{3}, \lambda_{4}\right\}\right\rangle
$$

changes from symmetric, with

$$
\left\langle\lambda_{1}+\lambda_{4}\right\rangle=\left\langle\lambda_{2}+\lambda_{3}\right\rangle=0
$$

to highly antisymmetric, as is shown in Table I. It is interesting that, within the statistical accuracy of the data, an approximate "pairing rule" [12] is satisfied, even away from equilibrium:

$$
\left\langle\lambda_{1}+\lambda_{4}\right\rangle \dot{=}\left\langle\lambda_{2}+\lambda_{3}\right\rangle \dot{=}-\dot{S} / 2 k
$$

Even at equilibrium the time variation of the local exponents is quite wild, with frequent crossings and reorderings, as is shown in Figures 5 and 6 . The information dimensions corresponding to the long-time-averaged spectra, calculated as suggested by Kaplan and Yorke, are also given in the Table. They indicate a decrease in dimensionality from 4 (at $\varepsilon=0.00$ ) to near 3 (at $\varepsilon=0.2631$ ), followed by collapse to 1 for larger values of the temperature gradient. 

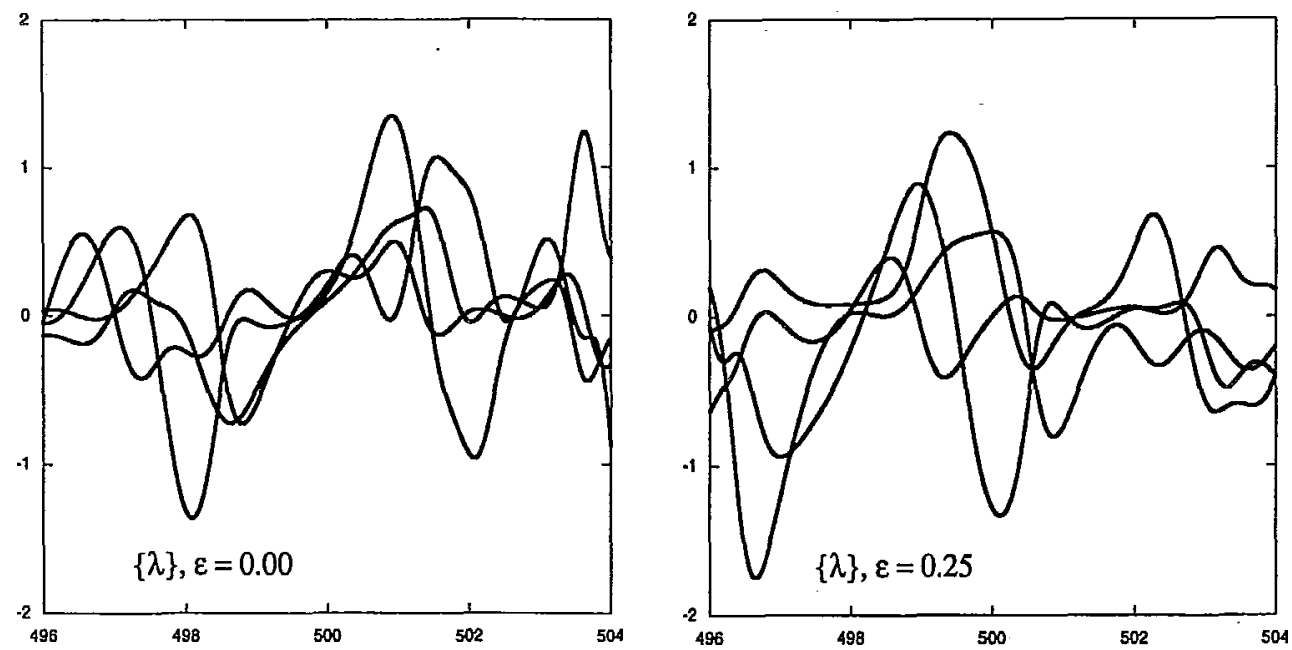

Fig. 5. Variation with time of the local (instantaneous) Lyapunov exponents with time for the same trajectory segments illustrated in Figure 2. Note the repeated crossings of the various exponents and the wide range of values which they cover. All 16 sign combinations and all 24 possible orderings of the local exponents occur in a longer segment.
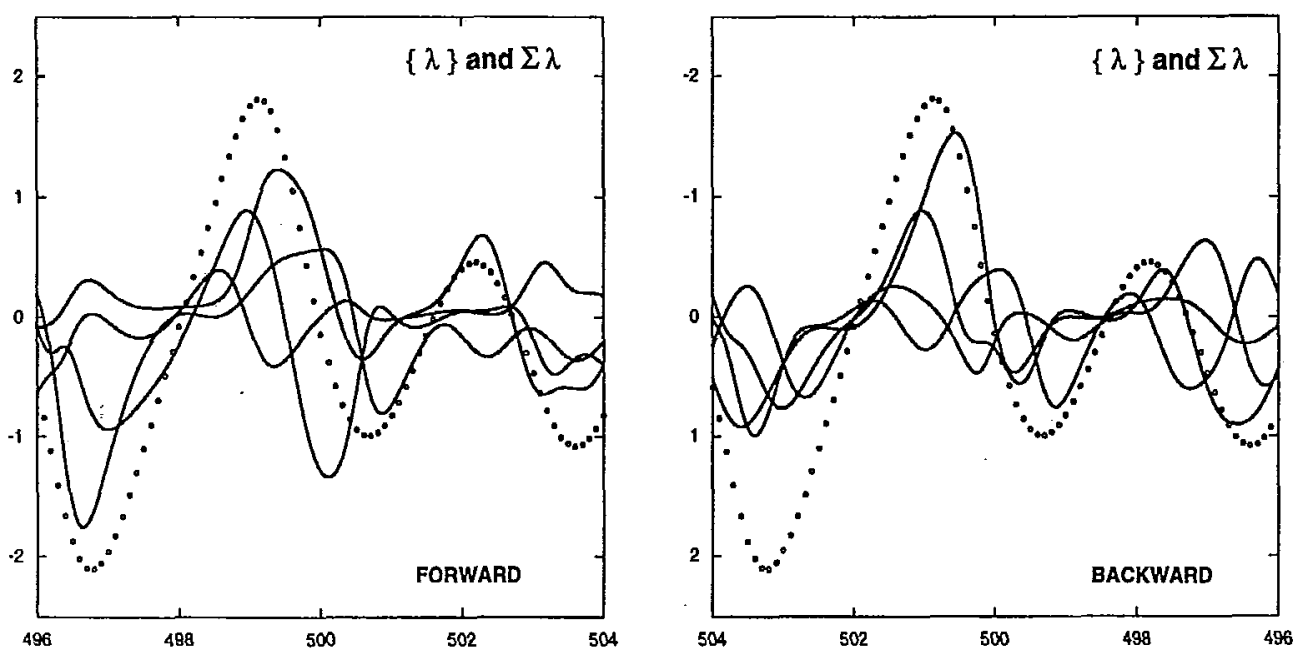

Fig. 6. Variation of the local (instantaneous) Lyapunov exponents and their sums with time for forward and backward trajectory segments. Notice that although there is no simple relation between the individual exponents in the forward and backward directions the simple sum rule, $\sum \lambda \equiv \xi+\xi$ requires that the sums (open circles in the plot) in the forward and backward directions simply change sign. The time symmetry of the motion is broken by Lyapunov instability, which reflects the past history, but not the future 
Table I. The long-time-averaged Lyapunov exponents, the corresponding information dimensions $D_{K Y}$, and the external entropy production rate $S$ are given as functions of the maximum temperature gradient $\varepsilon$. Fourth-order Runge-Kutta integration, with one billion timesteps of 0.001 , was used for these simulations

\begin{tabular}{ccccccc}
\hline$\varepsilon$ & $\left\langle\lambda_{1}\right\rangle$ & $\left\langle\lambda_{2}\right\rangle$ & $\left\langle\lambda_{3}\right\rangle$ & $\left\langle\lambda_{4}\right\rangle$ & $D_{K Y}$ & $\dot{S} / k$ \\
\hline 0.00 & +0.067 & +0.002 & -0.002 & -0.067 & 4.00 & 0.000 \\
0.05 & +0.066 & +0.002 & -0.002 & -0.066 & 3.99 & 0.001 \\
0.10 & +0.065 & +0.002 & -0.002 & -0.067 & 3.96 & 0.003 \\
0.15 & +0.065 & +0.001 & -0.004 & -0.068 & 3.91 & 0.006 \\
0.20 & +0.069 & +0.000 & -0.016 & -0.085 & 3.62 & 0.032 \\
0.25 & +0.053 & +0.000 & -0.034 & -0.086 & 3.27 & 0.068 \\
0.30 & +0.000 & -0.040 & -0.040 & -0.053 & 1.00 & 0.133 \\
\hline
\end{tabular}

\section{TIME REVERS I B ILITY}

The thermally-driven oscillator's motion equations are precisely time-reversible. That is, any sequence of values $\{+q,+p,+\zeta,+\xi\}$ generated at the times $\{0, d t, 2 d t, 3 d t, \ldots t\}$ corresponds to a reversed solution, $\{+q,-p,-\xi,-\xi\}$ of the same motion equations, provided that the time-ordering of the sequence is reversed too. Thisformal time reversibility extends to the local and global Lyapunov exponents. Any time history of the offset vectors $\{\delta\}$ corresponds also to a solution of the same equations, $\{\delta=D \cdot \delta\}$ provided that the $p, \zeta$ and $\xi$ components of the offset vectors are changed in sign and the time ordering of their histories is reversed. In this formal implementation of time reversibility the entire spectrum of coefficients changes sign: $\{+\lambda\}$ $\rightarrow\{-\lambda\}$.

This time-symmetry property is readily confirmed numerically in the case of Hamiltonian flows. After a relatively short time an arbitrary initial choice for the vectors $\{\delta\}$ is rotated so as to agree with the principal axes of the flow. Once this alignment has occured, the symmetry of the Lyapunov spectrum is also achieved, with each positive local exponent paired with its negative counterpart. Away from equilibrium this symmetry is typically broken, as is demonstrated in Figure 6. To show this we consider the local Lyapunov exponents near the middle of a million timestep simulation. With the complete $\{+q,+p,+\xi,+\xi\}$ trajectory stored, it is possible to reverse the time ordering of the reversed points $\{+q,-p,-\xi,-\xi\}$ to study the Lyapunov spectrum of the time-reversed trajectory. The result is shown in Figure 6. The summed spectrum reverses perfectly while the individual exponents do not.

\section{CONCLUSIONS}

We believe that the oscillator studied here is the simplest conceivable representative of smooth time-reversible nonequilibrium systems. The behavior of this simple system is complex. Both the stationary analysis of the dynamical matrix $D$ and the comoving analysis of the local Lyapunov exponents $\{\lambda\}$ indicate expanding and contracting regions quite inconsistent with the simpler fixed-point notions of unstable and stable manifolds. Further, the matrix approach indicates an intricate distribution of phase-space singularities frustrating the usual approaches. The 
local Lyapunov spectrum has promise. It quantifies the difference between the forward and backward time directions described by the Second Law of Thermodynamics. At the same time the local Lyapunov exponents are themselves highly singular functions in space, despite their smooth variation in time along individual trajectories.

\section{Acknowledgments}

We thank Christoph Dellago (University of Rochester) as well as Janka Petravic, Dennis Isbister, and Fei Zhang (Australian Defence Force Academy) for pertinent comments and suggestions. We also had useful conversations with Denis Evans and Lamberto Rondoni at the Australian National University. Publication of this work was delayed for nearly a year, to allow for an extended correspondence with Dr. Politi (Italy). A part of this work was carried out at the Technical University of Berlin with the kind support of Siegfried Hess and Martin Schön. Work in Peter Raboin's Methods Development Group at the Lawrence Livermore National Laboratoiy was performed under the auspices of the United States Department of Energy through University of California Contract W-7405-Eng-48. The work was additionally supported by a giant to HAP from the Fonds zur Förderung der wissenschaftlichen Forschung, Grant PI 1428-PHY.

\section{References}

[1] S. Wiggins, Introduction to Applied Nonlinear Dynamical Systems and Chaos, Springer-Verlag, New York, 1990.

[2] Wm. G. Hoover, Time Reversibility, Computer Simulation, and Chaos, World Scientific, Singapore, 1999.

[3] Wm. G. Hoover, Computational Statistical Mechanics, Elsevier, New York, 1991.

[4] H. A. Posch and Wm. G. Hoover, Phys. Rev., E55, 6803 (1997).

[5] Ch. Dellago and Wm. G. Hoover, Phys. Lett., A268, 330 (2000).

[6] Wm. G. Hoover, C. G. Hoover, and D. J. Isbister, Chaos, Ergodic Convergence, and Fractal Instabilityfor a Thermostated Canonical Harmonic Oscillator, Phys. Rev. E 63, 026209 (2000).

[7] P. Gaspard, Chaos, Scattering, and Statistical Mechanics, Cambridge University Press, 1998. It is a pleasure to thank Rainer Klages and Pierre Gaspard for their interesting comments on this point.

[8] See both the articles and the discussion remarks in the two useful summary volumes: Microscopic Simulations of Complex Flows, ed. M. Mareschal, Plenum, New York, 1989 and Microscopic Simulations of Complex Hydrodynamic Phenomena, ed. M. Mareschal and B. L. Holian, Plenum, New York, 1992.

[9] D. J. Evans, E. G. D. Cohen, G. P. Morriss, Phys. Rev. Lett., 71, 2401 (1993). We are indebted to Denis Evans for this estimate of the relaxation time.

[10] G. 1. Martyna, M. L. Klein, and M. E. Tuckerman, J. Chem. Phys., 97, 2635 (1992).

[11] Numerical methods were first developed by Giancarlo Benettin's group, following related work by Spotswood Stoddard and Joseph Ford.

[12] D. J. Evans and G. P. Morriss, Statistical Mechanics of Nonequilibrium Liquids, Academic, New York, 1990. 